\title{
PRECISE AND FAST COMPUTATION OF THE GENERAL COMPLETE ELLIPTIC INTEGRAL OF THE SECOND KIND
}

\author{
TOSHIO FUKUSHIMA
}

\begin{abstract}
We developed an efficient procedure to evaluate two auxiliary complete elliptic integrals of the second kind $B(m)$ and $D(m)$ by using their Taylor series expansions, the definition of Jacobi's nome, and Legendre's relation. The developed procedure is more precise than the existing ones in the sense that the maximum relative errors are 1-3 machine epsilons, and it runs drastically faster; around 5 times faster than Bulirsch's cel2 and 16 times faster than Carlson's $R_{F}$ and $R_{D}$.
\end{abstract}

\section{INTRODUCTION}

1.1. Complete elliptic integrals of first and second kind. The complete elliptic integrals of the first and the second kind $K(m)$ and $E(m)$ appear in various fields of mathematical physics and engineering [1, 6]. Consult with the references of our recent work [17] for practical examples in astrophysics and celestial mechanics such as the expression of gravitational potential and the acceleration vector caused by a uniform ring. The integrals are defined as

$$
K(m) \equiv \int_{0}^{\pi / 2} \frac{d \theta}{\sqrt{1-m \sin ^{2} \theta}}, E(m) \equiv \int_{0}^{\pi / 2}\left(\sqrt{1-m \sin ^{2} \theta}\right) d \theta .
$$

They are the special value of the incomplete elliptic integrals of the first and the second kind, $F(\varphi \mid m)$ and $E(\varphi \mid m)$ as

$$
K(m)=F\left(\frac{\pi}{2} \mid m\right), \quad E(m)=E\left(\frac{\pi}{2} \mid m\right),
$$

where the incomplete integrals are defined as

$$
F(\varphi \mid m) \equiv \int_{0}^{\varphi} \frac{d \theta}{\sqrt{1-m \sin ^{2} \theta}}, E(\varphi \mid m) \equiv \int_{0}^{\varphi}\left(\sqrt{1-m \sin ^{2} \theta}\right) d \theta .
$$

Refer to the formulas $110.02,110.03,110.06$, and 110.07 of [6]. We frequently cite the formulas of this handbook throughout the present article. Then we abbreviate the references to its formulas as BF110.02 hereafter. The complete integrals are real-valued when $m \leq 1$. Their special values are

$$
K(0)=E(0)=\frac{\pi}{2}, K(1)=+\infty, E(1)=1 .
$$

Received by the editor February 10, 2010 and, in revised form, April 22, 2010.

2010 Mathematics Subject Classification. Primary 33E05.

Key words and phrases. Elliptic integral. 
The values for negative parameters are reduced to those for positive ones as

$$
K(m)=\frac{K\left(m_{r}\right)}{\sqrt{m_{c}}}, \quad E(m)=\sqrt{m_{c}} E\left(m_{r}\right), \quad(m<0)
$$

where

$$
m_{c} \equiv 1-m, \quad m_{r} \equiv \frac{-m}{1-m},
$$

are the complementary and the reduced parameters, respectively. The latter satisfies the condition $0<m_{r}<1$ when $m<0$. These transformation formulas are obtained as the special cases of the imaginary modulus transformation formulas of the incomplete integrals. Refer to the first and second forms of BF160.02. These features enable us to consider only the cases when $0<m<1$.

1.2. Auxiliary complete elliptic integrals. Rather important are not the pair of $K(m)$ and $E(m)$ but a pair of auxiliary complete elliptic integrals of the second kind [2]:

$$
B(m) \equiv \int_{0}^{\pi / 2} \frac{\cos ^{2} \theta d \theta}{\sqrt{1-m \sin ^{2} \theta}}, \quad D(m) \equiv \int_{0}^{\pi / 2} \frac{\sin ^{2} \theta d \theta}{\sqrt{1-m \sin ^{2} \theta}} .
$$

They are linearly related with $K(m)$ and $E(m)$ as

$$
K(m)=B(m)+D(m), \quad E(m)=B(m)+m_{c} D(m) .
$$

The special values are computed as

$$
B(0)=D(0)=\frac{\pi}{4}, \quad B(1)=1, \quad D(1)=+\infty .
$$

The values for negative parameters are alternatively reduced to those for positive ones as

$$
B(m)=\frac{D\left(m_{r}\right)}{\sqrt{m_{c}}}, \quad D(m)=\frac{B\left(m_{r}\right)}{\sqrt{m_{c}}}(m<0) .
$$

These are derived from equations (1.5) and (1.8). Therefore we can restrict ourselves to the case $0<m<1$. The auxiliary integrals are needed in computing the derivatives of $K(m)$ and $E(m)$ without cancellation as

$$
\frac{d K(m)}{d m}=\frac{B(m)}{2 m_{c}}, \frac{d E(m)}{d m}=\frac{-D(m)}{2} .
$$

These are derived from BF710.00 and BF710.02. Also, $B(m)$ or $D(m)$ is required in the evaluation of the corresponding incomplete integrals:

$$
B(\varphi \mid m) \equiv \int_{0}^{\varphi} \frac{\cos ^{2} \theta d \theta}{\sqrt{1-m \sin ^{2} \theta}}, \quad D(\varphi \mid m) \equiv \int_{0}^{\varphi} \frac{\sin ^{2} \theta d \theta}{\sqrt{1-m \sin ^{2} \theta}} .
$$

This is because their computations for arbitrary value of $\varphi$ are transformed to those for the case $0<\varphi<\pi / 2$ by the amplitude reduction formulas

$$
B(\varphi \pm j \pi \mid m)=B(\varphi \mid m) \pm 2 j B(m), \quad D(\varphi \pm j \pi \mid m)=D(\varphi \mid m) \pm 2 j D(m) .
$$

These are derived from those of $F(\varphi \mid m)$ and $E(\varphi \mid m)$ given in BF113.02. 
1.3. Auxiliary incomplete elliptic integrals. The auxiliary incomplete elliptic integrals are required in computing the partial derivatives of $F(\varphi \mid m)$ and $E(\varphi \mid m)$ with respect to the parameter $m$ without cancellation as

$$
\begin{gathered}
\left(\frac{\partial F(\varphi \mid m)}{\partial m}\right)_{\varphi}=\frac{1}{2 m_{c}}\left[B(\varphi \mid m)-\left(\frac{\sin \varphi \cos \varphi}{\sqrt{1-m \sin ^{2} \varphi}}\right)\right], \\
\left(\frac{\partial E(\varphi \mid m)}{\partial m}\right)_{\varphi}=\frac{-D(\varphi \mid m)}{2} .
\end{gathered}
$$

Refer to BF710.07 and BF710.09. The former derivative is related with the partial derivatives of Jacobian elliptic functions with respect to $m$ as

$$
\begin{gathered}
\left(\frac{\partial \operatorname{sn}(u \mid m)}{\partial m}\right)_{u}=\operatorname{cn}(u \mid m) \operatorname{dn}(u \mid m)\left(\frac{\partial F(\varphi \mid m)}{\partial m}\right)_{\varphi} \\
\left(\frac{\partial \operatorname{cn}(u \mid m)}{\partial m}\right)_{u}=-\operatorname{sn}(u \mid m) \operatorname{dn}(u \mid m)\left(\frac{\partial F(\varphi \mid m)}{\partial m}\right)_{\varphi} \\
\left(\frac{\partial \operatorname{dn}(u \mid m)}{\partial m}\right)_{u}=-m \operatorname{sn}(u \mid m) \operatorname{cn}(u \mid m)\left(\frac{\partial F(\varphi \mid m)}{\partial m}\right)_{\varphi}, \\
\left(\frac{\partial \operatorname{am}(u \mid m)}{\partial m}\right)_{u}=\operatorname{dn}(u \mid m)\left(\frac{\partial F(\varphi \mid m)}{\partial m}\right)_{\varphi} .
\end{gathered}
$$

Refer to BF710.50 through BF710.53. These derivatives are necessary in applying the method of variation of constant to the perturbed rotational motion of a rigid body [14, 15].

1.4. Cancellation problem. One may claim that the combination of $K(m)$ and $E(m)$ is sufficient for the purposes discussed in the previous subsection. Either $B(m)$ or $D(m)$ is computable from the pair of $K(m)$ and $E(m)$ as

$$
B(m)=\frac{E(m)-m_{c} K(m)}{m}, D(m)=\frac{K(m)-E(m)}{m} .
$$

These are obtained by solving equation (1.8) with respect to $B(m)$ and $D(m)$. Nevertheless, these expressions face a severe loss of information in numerical computation. This is true especially when $m$ is small. Such a situation frequently occurs when we discuss the rotational motions of rigid bodies under weak torques. Good examples are those of solid planets, satellites, and asteroids in the solar system. The typical values of $m$ is of the order of $10^{-7}$ in these cases [13. Figure 1 shows the parameter dependence of the logarithms of relative errors of $B(m)$ computed by two methods: equation (1.20) using the given values of $K(m)$ and $E(m)$ and Bulirsch's cel2 2]. Here the function cel2 is defined as

$$
\operatorname{cel2}\left(k_{c}, a, b\right) \equiv \int_{0}^{\infty}\left(\frac{a+b \xi^{2}}{\left(1+\xi^{2}\right) \sqrt{\left(1+\xi^{2}\right)\left(1+k_{c}^{2} \xi^{2}\right)}}\right) d \xi .
$$

It can directly evaluate $B(m)$ and $D(m)$ as

$$
B(m)=\operatorname{cel} 2\left(k_{c}, 1,0\right), \quad D(m)=\operatorname{cel} 2\left(k_{c}, 0,1\right),
$$

where

$$
k_{c} \equiv \sqrt{m_{c}}
$$




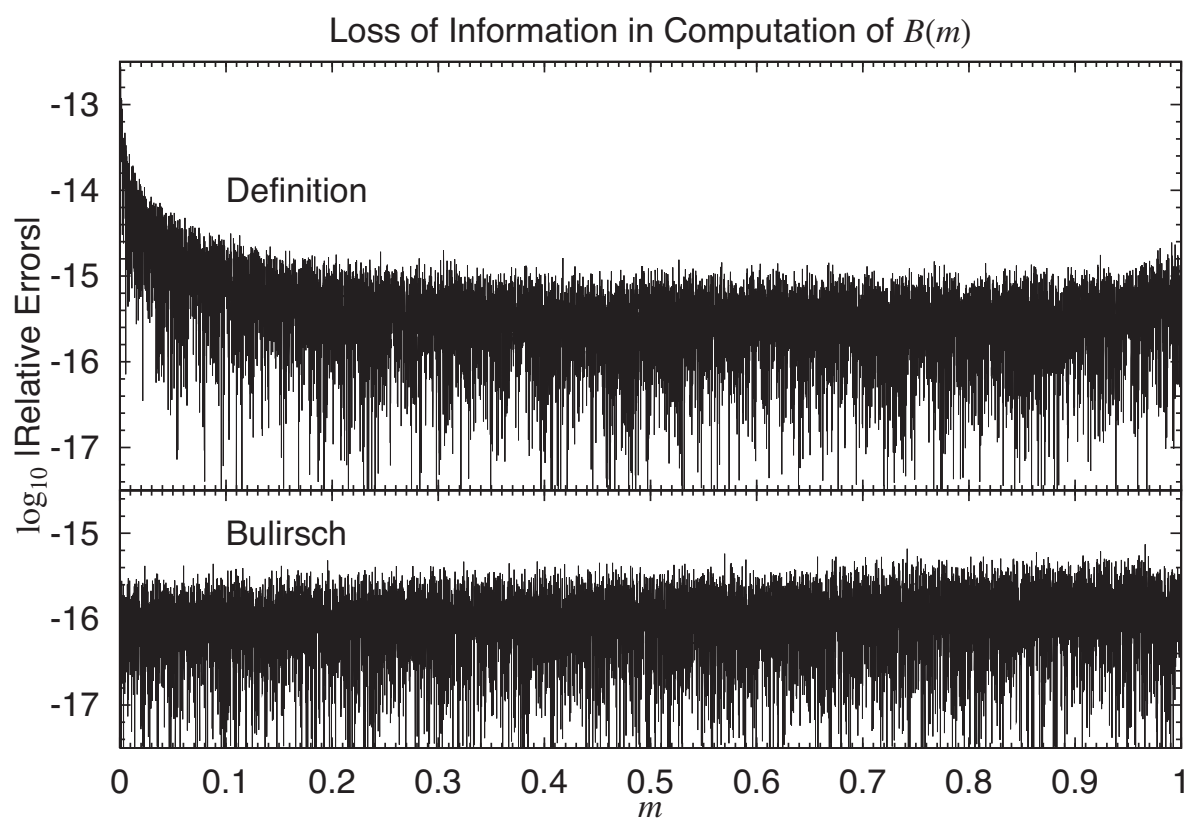

Figure 1. Loss of Information in Computation of $B(m)$. Shown are the relative errors of two methods to compute $B(m)$ in the double precision environment; the defining formula given in equation (1.20) and Bulirsch's cel2.

is the complementary modulus. The errors shown in the figure are measured as the differences from the quadruple precision computations prepared by qcel2. It is the quadruple precision extension of cel2. The accuracy of the quadruple precision computation is confirmed at the level of $10^{-33}$. This was done by random comparison with the extremely high precision computation in 40 digits by Mathematica 22. We computed the values of $K(m)$ and $E(m)$ by Cody's Chebyshev approximate formulas of Hastings type [20, 10, 11, 12] in preparing the graph of the former method. We confirm that the manner of round-off error increase is independent on the procedure to compute $K(m)$ and $E(m)$. A similar result as shown in Figure 1 is obtained for $D(m)$. The reason of the significant increase of round-off errors in case of the defining formulas is clear. This is because equation (1.20) is of the form of the difference of two positive quantities of similar magnitude when $m \sim 0$. Figure 2 illustrates the parameter dependence of $K(m), E(m), B(m)$, and $D(m)$. It is obvious that $m_{c} \sim 1$ when $m$ is small. Then $E(m)$ and $m_{c} K(m)$ become of a similar magnitude. This produces round-off errors in evaluating $B(m)$. Similarly, $K(m)$ and $E(m)$ are roughly the same when $m \sim 0$. This is the cause of information loss in computing $D(m)$. 


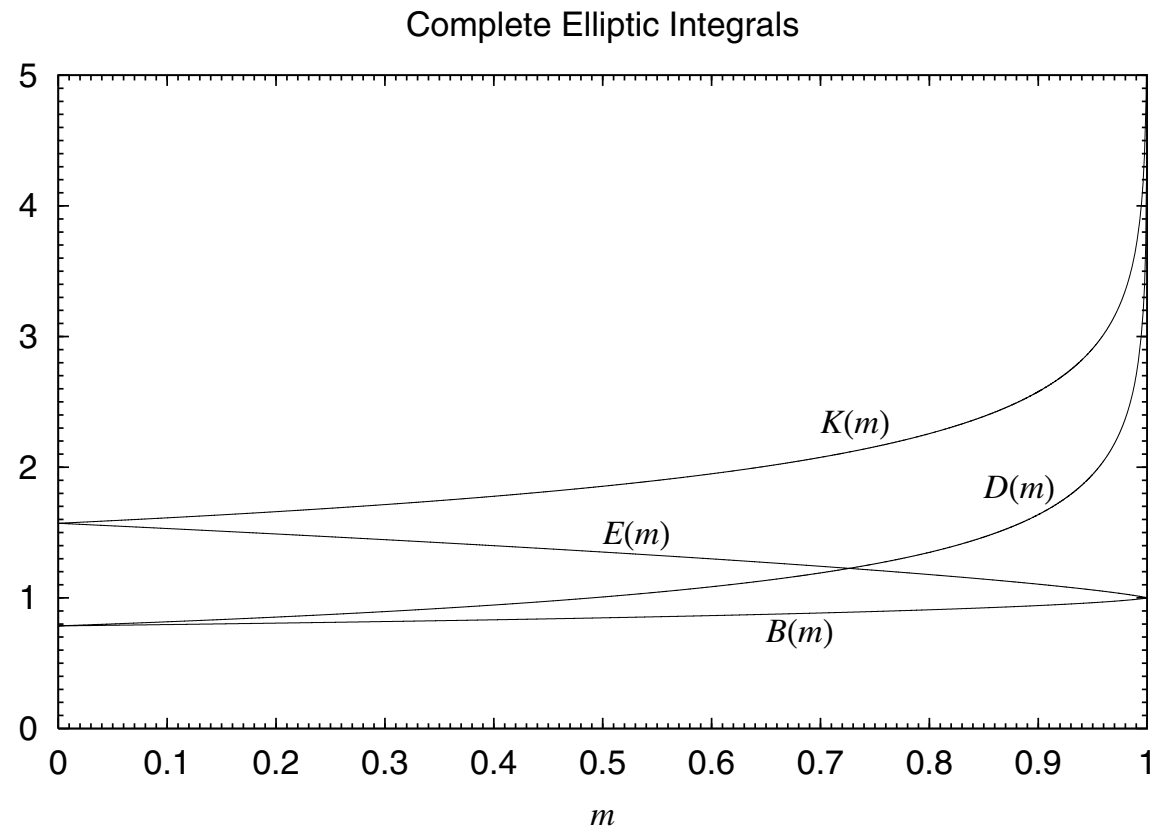

Figure 2. Parameter Dependence of Complete Elliptic Integrals.

1.5. Comparison of existing methods. We need specific procedures in order to compute $B(m)$ and/or $D(m)$ with ignorable round-off errors. There exist two methods: Bulirsch's cel2 described already and Carlson's $R_{F}$ and $R_{D}[7,8$. The latter functions are defined as

$$
\begin{aligned}
R_{F}(x, y, z) & \equiv \frac{1}{2} \int_{0}^{\infty} \frac{d t}{\sqrt{(t+x)(t+y)(t+z)}}, \\
R_{D}(x, y, z) & \equiv \frac{3}{2} \int_{0}^{\infty} \frac{d t}{\sqrt{(t+x)^{3}(t+y)(t+z)}},
\end{aligned}
$$

and related with $F(\varphi \mid m)$ and $D(\varphi \mid m)$ as

$$
\begin{gathered}
F(\varphi \mid m)=\sin \varphi R_{F}\left(\cos ^{2} \varphi, 1-m \sin ^{2} \varphi, 1\right), \\
D(\varphi \mid m)=\left(\frac{\sin ^{3} \varphi}{3}\right) R_{D}\left(\cos ^{2} \varphi, 1-m \sin ^{2} \varphi, 1\right) .
\end{gathered}
$$

Therefore, we can use them in computing $B(m)$ and $D(m)$ as

$$
B(m)=K(m)-D(m), \quad K(m)=R_{F}\left(0, m_{c}, 1\right), \quad D(m)=\frac{R_{D}\left(0, m_{c}, 1\right)}{3} .
$$

Numerical comparison with the higher precision computations revealed that both of these methods are almost always precise. The exception is the case of $B(m)$ computed by Carlson's functions if $m \sim 1$. Figures 3 and 4 depict the parameter dependence of the relative errors of the two methods to compute $B(m)$ and $D(m)$ in the double precision environment. The precision loss of $B(m)$ in Carlson's case when $m \sim 1$ is due to its expression shown in the above. There $B(m)$ is expressed as the difference of two positive quantities $K(m)$ and $D(m)$. Both $K(m)$ and $D(m)$ 


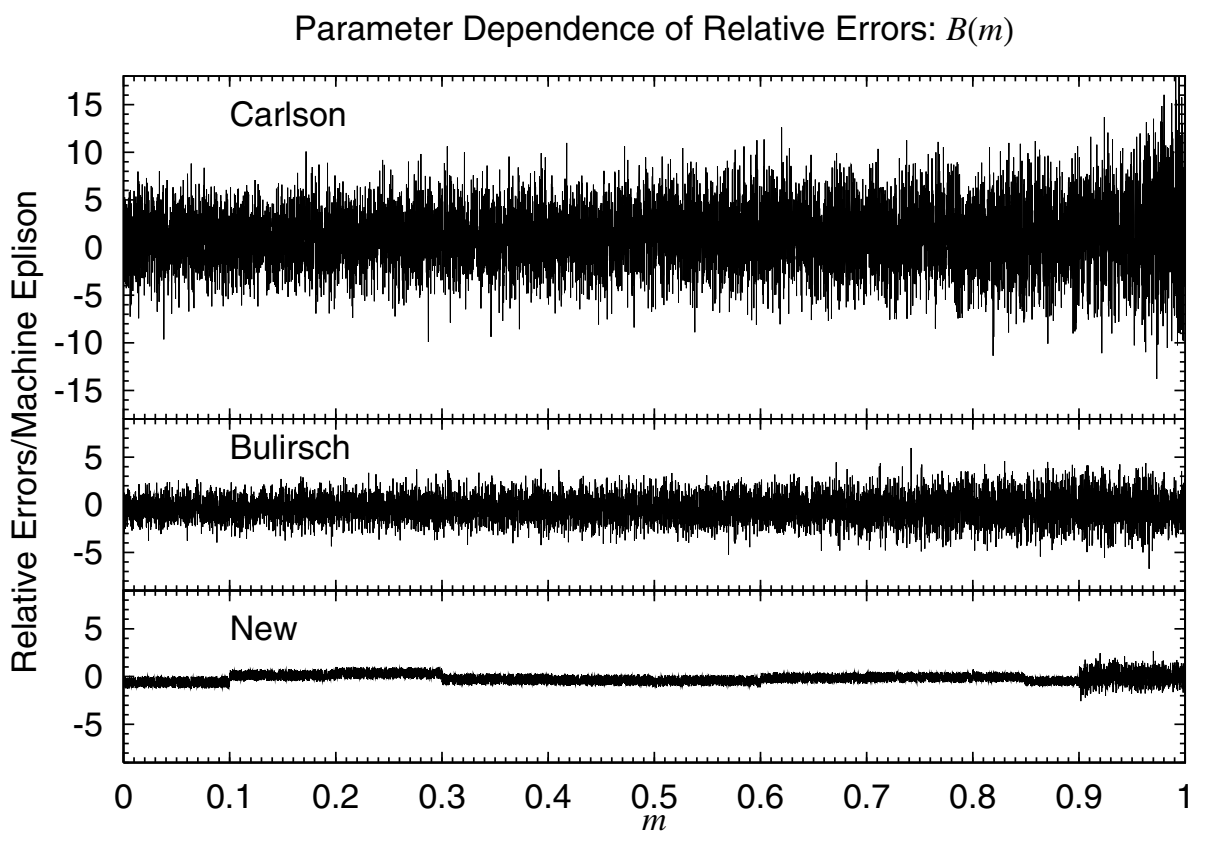

Figure 3. Parameter Dependence of Relative Errors of $B(m)$. Shown are the relative errors of three methods to compute $B(m)$ in the double precision environment; Carlson's $R_{F}$ and $R_{D}$, Bulirsch's cel2, and the new method presented in $₫ 2$.

go to infinity logarithmically when $m \rightarrow 1$. See Figure 2 again. Then the magnitude of $K(m)$ and $D(m)$ become similar. Thus Carlson's form faces a cancellation. This means that cel2 is more appropriate than $R_{F}$ and $R_{D}$ in the sense of computational precision. Table 1 lists the averaged CPU time of these methods. We measured the CPU times as simple means of the results for $2^{24}-1$ equally spaced grid points in the standard domain of the parameter $0<m<1$ and scaled by that to compute the sine function in the double precision environment. All the computation codes were written in Fortran 77/90, compiled by the Intel Visual Fortran 8.0, and executed at a PC with Intel Core Duo processor under Windows XP. The table clearly shows that cel2 runs 2.9-3.5 times faster than the combination of $R_{F}$ and $R_{D}$. This mainly owes to the difference in the speed of convergence of the main algorithm. Bulirsch's cel2 is based on the Landen transformation in combination with the arithmetic-geometric mean [2, 3, 4, 5]. It is of quadratic convergence. Namely the number of significant digits is doubled by each iteration. Meanwhile Carlson's functions rely on the duplication theorem [7, 8]. It is of linear convergence. Then the number of significant digits increases by a same amount in each application. Thus the higher the desired relative precision is, the faster cel2 becomes than $R_{F}$ and $R_{D}$. This is observed from the ratios of their CPU times in the single and double precision environments. Therefore, cel2 is more preferable than the combination of $R_{F}$ and $R_{C}$ also in terms of the computational speed. Actually, cel2 runs around three times faster than the pair of $R_{F}$ and $R_{C}$ already in the single precision environment. Nevertheless, it is also true that even cel2 requires 
Parameter Dependence of Relative Errors: $D(m)$

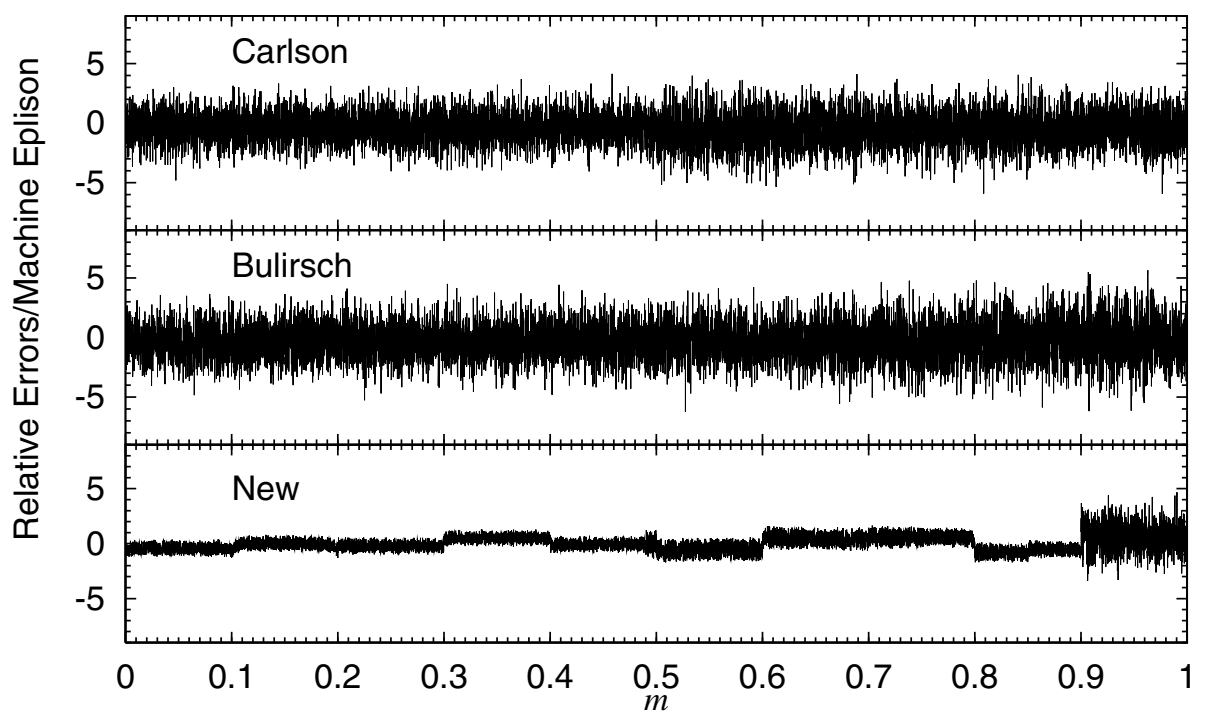

Figure 4. Parameter Dependence of Relative Errors of $D(m)$. Same as Figure 3 but for $D(m)$.

TABle 1. Averaged CPU Times to Compute $B(m)$ and $D(m)$ Simultaneously. The unit of CPU time is that to compute the double precision sine function.

\begin{tabular}{llrr}
\hline Method & Procedures & Single & Double \\
\hline Carlson & $R_{F} \& R_{D}$ & 18.0 & 53.5 \\
Bulirsch & cel2 & 6.2 & 15.5 \\
New & elbd & 1.1 & 3.2 \\
\hline
\end{tabular}

a significant amount of computational time, say 6-16 times more than that of the sine function. This is still a large computational labor as we consider the frequent needs to evaluate the integrals in practical applications.

1.6. Introduction of new method. Recently we developed a new method to compute $K(m)$ and/or $E(m)$ in [17. Hereafter, we cite it as Paper I. The key techniques used there are the utilization of Taylor series expansion and the combination of the defining relation of Jacobi's nome and Legendre's relation. This is a continuation of our trials to accelerate the procedures to compute the complete and incomplete elliptic integrals and the Jacobian elliptic functions [19, 16, 17, 18. The new method is sufficiently precise and significantly faster than the existing procedures to compute $K(m)$ and/or $E(m)$ including Cody's method as well as Innes' classic formulation [21. None of the procedures discussed in Paper I including ours is not suitable to compute $B(m)$ and/or $D(m)$ as we saw in Figure 1. Then we 
TABle 2. Necessary Minimum Order of Taylor Expansion Polynomials of $B(m)$ and $D(m)$

\begin{tabular}{crrrr}
\hline & \multicolumn{2}{c}{ Single } & \multicolumn{2}{c}{ Double } \\
$m$ & $J_{B}$ & $J_{D}$ & $J_{B}$ & $J_{D}$ \\
\hline$(0.0,0.1]$ & 5 & 5 & 11 & 11 \\
$(0.1,0.2]$ & 5 & 5 & 11 & 11 \\
$(0.2,0.3]$ & 6 & 6 & 12 & 12 \\
$(0.3,0.4]$ & 6 & 6 & 12 & 13 \\
$(0.4,0.5]$ & 6 & 7 & 12 & 15 \\
$(0.5,0.6]$ & 6 & 8 & 13 & 16 \\
$(0.6,0.7]$ & 7 & 8 & 15 & 17 \\
$(0.7,0.8]$ & 9 & 10 & 18 & 20 \\
$(0.8,0.85]$ & 7 & 8 & 14 & 17 \\
$(0.85,0.9]$ & 9 & 10 & 18 & 20 \\
\hline
\end{tabular}

adapt our approach in Paper I to the computation of $B(m)$ and $D(m)$. The resulting method is significantly more precise than the existing procedures. See Figures 3 and 4 again. The errors of the new method are 1-3 machine epsilons at most. Meanwhile, the new method runs much faster than cel2. See Table 1 again. In this short article, we will present the detail of the new method to compute $B(m)$ and $D(m)$ simultaneously.

\section{NEW METHOD}

Let us consider computing the auxiliary complete elliptic integrals $B(m)$ and $D(m)$. Refer to Paper I for the basic background of the present approach. Once the auxiliary integrals are computed, we can calculate $K(m)$ and $E(m)$ from them without cancellation by using equation (1.8).

2.1. Case of small parameter. When $0<m \leq 0.9$, we evaluate $B(m)$ and $D(m)$ by their piecewise approximate polynomials derived from their Taylor series expansions. In constructing the piecewise polynomials, we divide the given domain $(0.0,0.9]$ into 10 subdomains; $(0.0,0.1],(0.1,0.2],(0.2,0.3],(0.3,0.4],(0.4,0.5]$, $(0.5,0.6],(0.6,0.7],(0.7,0.8],(0.8,0.85]$, and $(0.85,0.9]$. In each subdomain, the approximate polynomials are written as

$$
B(m) \approx \sum_{j=0}^{J_{B}} B_{j}\left(m-m_{0}\right)^{j}, \quad D(m) \approx \sum_{j=0}^{J_{D}} D_{j}\left(m-m_{0}\right)^{j} .
$$

The necessary minimum order of the polynomials $J_{B}$ and $J_{D}$ are listed in Table 2 for the single and double precision environment. We obtained them by numerical comparison with the quadruple precision computations. We set the reference value of $m$ in each subdomain $m_{0}$ as the center values of the corresponding subdomains. Namely, they are $0.05,0.15,0.25,0.35,0.45,0.55,0.65,0.75,0.825$, and 0.875 . Meanwhile, the coefficients $B_{j}$ and $D_{j}$ themselves are explicitly given in Tables 3 through 12. We obtained them by Mathematica 22] with a command like

$$
\text { Series }[(\text { EllipticE[m] }-(1-\mathrm{m}) \text { EllipticK }[\mathrm{m}]) / \mathrm{m},\{\mathrm{m}, 0.05,12\}]]
$$

This gives the coefficients $B_{j}$ in Table 3 
Table 3. Coefficients of Taylor Expansion Polynomials of $B(m)$ and $D(m): 0.0<m \leq 0.1$. The coefficients are expressed with a few more digits than necessary in order to avoid round-off errors in the implementation.

\begin{tabular}{rcc}
\hline$j$ & $B_{j}$ & $D_{j}$ \\
\hline 0 & 0.790401413584395132 & 0.800602040206397048 \\
1 & 0.102006266220019155 & 0.313994477771767757 \\
2 & 0.039878395558551461 & 0.205913118705551955 \\
3 & 0.021737136375982167 & 0.157744346538923994 \\
4 & 0.013960979767622058 & 0.130595077319933092 \\
5 & 0.009892518822669142 & 0.113308474489758567 \\
6 & 0.007484612400663336 & 0.101454199173630195 \\
7 & 0.005934625664295474 & 0.092918784207297437 \\
8 & 0.004874249053581664 & 0.086565380148168087 \\
9 & 0.004114606930310886 & 0.081727984665103014 \\
10 & 0.003550452989196177 & 0.077990665729107038 \\
11 & 0.003119229959988475 & 0.075080426851268007 \\
\hline
\end{tabular}

TABle 4. Coefficients of Taylor Expansion Polynomials of $B(m)$ and $D(m): 0.1<m \leq 0.2$

\begin{tabular}{rcc}
\hline$j$ & $B_{j}$ & $D_{j}$ \\
\hline 0 & 0.801024064452844894 & 0.834232667811735098 \\
1 & 0.110695344529634015 & 0.360495281619098276 \\
2 & 0.047348746716993718 & 0.262379664114505869 \\
3 & 0.028484367255041423 & 0.223723944518094276 \\
4 & 0.020277811444003597 & 0.206447811775681053 \\
5 & 0.015965005853099119 & 0.199809440876486856 \\
6 & 0.013441320273553635 & 0.199667451603795275 \\
7 & 0.011871565736951440 & 0.204157558868236842 \\
8 & 0.010868363672485521 & 0.212387467960572375 \\
9 & 0.010231587232710565 & 0.223948914061499360 \\
10 & 0.009849585546666211 & 0.238708097425597860 \\
11 & 0.009656606347153765 & 0.256707203545463756 \\
\hline
\end{tabular}


TABle 5. Coefficients of Taylor Expansion Polynomials of $B(m)$ and $D(m): 0.2<m \leq 0.3$

\begin{tabular}{rcc}
\hline$j$ & $B_{j}$ & $D_{j}$ \\
\hline 0 & 0.812597772919920493 & 0.873152581892675550 \\
1 & 0.121109617945510113 & 0.420622230667770216 \\
2 & 0.057293376831239877 & 0.344231061559450379 \\
3 & 0.038509451602167328 & 0.331133021818721762 \\
4 & 0.030783430301775233 & 0.345277285052808412 \\
5 & 0.027290564934732527 & 0.377945322150393392 \\
6 & 0.025916369289445199 & 0.427378012464553881 \\
7 & 0.025847203343361799 & 0.494671744307822406 \\
8 & 0.026740923539348855 & 0.582685115665646201 \\
9 & 0.028464314554825705 & 0.695799207728083165 \\
10 & 0.030995446237278954 & 0.840018401472533403 \\
11 & 0.034384369179940976 & 1.023268503573606061 \\
12 & 0.038738002072493936 & 1.255859085136282496 \\
\hline
\end{tabular}

Table 6. Coefficients of Taylor Expansion Polynomials of $B(m)$ and $D(m): 0.3<m \leq 0.4$

\begin{tabular}{rcc}
\hline$j$ & $B_{j}$ & $D_{j}$ \\
\hline 0 & 0.825323557983515895 & 0.919027039242097348 \\
1 & 0.133862116083687790 & 0.501002159288247514 \\
2 & 0.071011293597988675 & 0.468831270566456863 \\
3 & 0.054178477417387376 & 0.517714227776400015 \\
4 & 0.049451744948102993 & 0.620843391317303107 \\
5 & 0.050222196224107476 & 0.782364393786869723 \\
6 & 0.054742913171830353 & 1.019114535076102913 \\
7 & 0.062746257927001699 & 1.359345202748496052 \\
8 & 0.074669881043476886 & 1.845717302358827942 \\
9 & 0.091480845177733472 & 2.541071703153920729 \\
10 & 0.114705092110997824 & 3.537404655208041337 \\
11 & 0.146571132581439876 & 4.969296002977425930 \\
12 & 0.190257137333846284 & 7.033822870030031126 \\
13 & & 10.02004322503447140 \\
\hline
\end{tabular}


TABle 7. Coefficients of Taylor Expansion Polynomials of $B(m)$ and $D(m): 0.4<m \leq 0.5$

\begin{tabular}{rcc}
\hline$j$ & $B_{j}$ & $D_{j}$ \\
\hline 0 & 0.839479570270612971 & 0.974404366546369673 \\
1 & 0.149916440306396336 & 0.613246805394160910 \\
2 & 0.090831935819428835 & 0.671096669502166996 \\
3 & 0.080347033483341786 & 0.870727620185086140 \\
4 & 0.085638440500470454 & 1.229542231202690761 \\
5 & 0.101954725932990372 & 1.826605967544420569 \\
6 & 0.130574811533616015 & 2.806934530997762740 \\
7 & 0.176105076358849928 & 4.418789329084028134 \\
8 & 0.246835164402955447 & 7.083236057478765325 \\
9 & 0.356424476867718855 & 11.51508812055758294 \\
10 & 0.527002562230102743 & 18.93151118599927464 \\
11 & 0.794389634259304750 & 31.41199693820496388 \\
12 & 1.216762532429718021 & 52.52072945457582854 \\
13 & & 88.38485473506529806 \\
14 & & 149.5663744939804784 \\
15 & & 254.3179084310411743 \\
\hline
\end{tabular}

Table 8. Coefficients of Taylor Expansion Polynomials of $B(m)$ and $D(m): 0.5<m \leq 0.6$

\begin{tabular}{rcc}
\hline$j$ & $B_{j}$ & $D_{j}$ \\
\hline 0 & 0.855469615156419991 & 1.043455295115133534 \\
1 & 0.170896072689739584 & 0.779625721928504850 \\
2 & 0.121335229026948226 & 1.029742360932067582 \\
3 & 0.128201883574947410 & 1.622037223411353130 \\
4 & 0.164687281451527560 & 2.787989531185347620 \\
5 & 0.237418908749381742 & 5.048381487372069147 \\
6 & 0.369208104716495452 & 9.463277611943484295 \\
7 & 0.605658733847927717 & 18.18148994942766790 \\
8 & 1.033705561557812744 & 35.58098059117916870 \\
9 & 1.818988489363267885 & 70.63393546191445013 \\
10 & 3.279377651273850938 & 141.8285800834330593 \\
11 & 6.029888380717536331 & 287.4487512501321663 \\
12 & 11.26979685557794172 & 587.1153846499230762 \\
13 & 21.35457785038283450 & 1207.065435225480616 \\
14 & & 2495.588727248664223 \\
15 & & 5184.692429394806441 \\
16 & & 10817.21333690413275 \\
\hline
\end{tabular}


TABle 9. Coefficients of Taylor Expansion Polynomials of $B(\mathrm{~m})$ and $D(m): 0.6<m \leq 0.7$

\begin{tabular}{rcc}
\hline$j$ & $B_{j}$ & $D_{j}$ \\
\hline 0 & 0.873920061848643136 & 1.133678336575733166 \\
1 & 0.199814057482376946 & 1.048643173729970391 \\
2 & 0.172769615878015213 & 1.753465041198464516 \\
3 & 0.228106913284202167 & 3.523182726803385513 \\
4 & 0.370468141118071220 & 7.749476413813974582 \\
5 & 0.679271252884820555 & 17.98645005585073306 \\
6 & 1.348008496681757302 & 43.25591634623261333 \\
7 & 2.827670976853820704 & 106.6815344540960170 \\
8 & 6.179468250123914084 & 268.0984865731174340 \\
9 & 13.93568601034281150 & 683.6241148502898048 \\
10 & 32.21892928105972203 & 1763.497085219187407 \\
11 & 76.00696295922610103 & 4592.374753831163809 \\
12 & 182.3214490877540696 & 12053.44101904888928 \\
13 & 443.5150764411264816 & 31846.66302074208170 \\
14 & 1091.854722902838829 & 84621.22135905680802 \\
15 & 2715.765866403819588 & 225956.4231829078900 \\
16 & & 605941.5172817588600 \\
17 & & 1631082.599539268321 \\
\hline
\end{tabular}

TABle 10. Coefficients of Taylor Expansion Polynomials of $B(m)$ and $D(m): 0.7<m \leq 0.8$

\begin{tabular}{rcc}
\hline$j$ & $B_{j}$ & $D_{j}$ \\
\hline 0 & 0.895902820924731621 & 1.260612826574911614 \\
1 & 0.243140003766786662 & 1.548665638082676581 \\
2 & 0.273081875594105532 & 3.553669411871607615 \\
3 & 0.486280007533573324 & 9.900444676104398756 \\
4 & 1.082747437228230918 & 30.32056661745247199 \\
5 & 2.743445290986452500 & 98.18025865888308915 \\
6 & 7.555817828670234627 & 329.7710104345570550 \\
7 & 22.05194082493752427 & 1136.655989742890393 \\
8 & 67.15640644740229408 & 3993.834335746229798 \\
9 & 211.2722537881770962 & 14242.72958655527085 \\
10 & 681.9037843053270682 & 51394.75729168872096 \\
11 & 2246.956231592536517 & 187246.7029146231521 \\
12 & 7531.483865999711792 & 687653.0923753899027 \\
13 & 25608.51260130241579 & 2542385.535653982270 \\
14 & 88140.74740089604971 & 9453781.219347490272 \\
15 & 306564.4242098446591 & 35328363.01797091708 \\
16 & 1076036.077811072194 & 132593262.3833930149 \\
17 & 3807218.502573632648 & 499544968.1840548215 \\
18 & 13566382.24422139551 & 1888409347.294438724 \\
19 & & 7160267534.478937192 \\
20 & & 27223307946.96339622 \\
\hline
\end{tabular}


TABle 11. Coefficients of Taylor Expansion Polynomials of $B(m)$ and $D(m): 0.8<m \leq 0.85$

\begin{tabular}{rcc}
\hline$j$ & $B_{j}$ & $D_{j}$ \\
\hline 0 & 0.915922052601931494 & 1.402200569110579095 \\
1 & 0.294714252429483394 & 2.322205897861749447 \\
2 & 0.435776709264636140 & 7.462158366466719683 \\
3 & 1.067328246493644239 & 29.43506890797307903 \\
4 & 3.327844118563268085 & 128.1590924337895775 \\
5 & 11.90406004445092906 & 591.0807036911982326 \\
6 & 46.47838820224626394 & 2830.546229607726377 \\
7 & 192.7556002578809477 & 13917.76431889392230 \\
8 & 835.3356299261900064 & 69786.10525163921228 \\
9 & 3743.124548343029103 & 355234.1420341879635 \\
10 & 17219.07731004063094 & 1830019.186413931054 \\
11 & 80904.60401669850158 & 9519610.812032515607 \\
12 & 386808.3292751742460 & 49920868.75574849454 \\
13 & 1876487.670110449342 & 263567700.9826023474 \\
14 & 9216559.908641567755 & 1399645765.120061119 \\
15 & & 7469935792.837635005 \\
16 & & 40041555958.35610574 \\
17 & & 215463066814.4966654 \\
\hline
\end{tabular}

TABle 12. Coefficients of Taylor Expansion Polynomials of $B(m)$ and $D(m): 0.85<m \leq 0.9$

\begin{tabular}{rcc}
\hline$j$ & $B_{j}$ & $D_{j}$ \\
\hline 0 & 0.931906061029524828 & 1.541690112721819084 \\
1 & 0.348448029538453861 & 3.379176214579645449 \\
2 & 0.666809178846938248 & 14.94058385670236672 \\
3 & 2.210769135708128663 & 81.91773929235074881 \\
4 & 9.491765048913406881 & 497.4900546551479866 \\
5 & 47.09304791027740853 & 3205.184010234846235 \\
6 & 255.9200460211233087 & 21457.32237355321926 \\
7 & 1480.029532675805408 & 147557.0156564174712 \\
8 & 8954.040904734313578 & 1035045.290185256525 \\
9 & 56052.48220982686950 & 7371922.334832212125 \\
10 & 360395.7241626000917 & 53143443.95142401142 \\
11 & 2367539.415273216078 & 386882347.5795976313 \\
12 & 15829949.57277684102 & 2839458401.528033778 \\
13 & 107415809.3278511100 & 20982661229.43898942 \\
14 & 738058546.0239595692 & 155961775401.7662418 \\
15 & 5126022002.555101497 & 1165096220419.884791 \\
16 & 35935340655.02416589 & 8742012983013.913805 \\
17 & 253988125761.2812212 & 65847254626723.66919 \\
18 & 1808180007145.359570 & 497679873706243.4393 \\
19 & & 3773018634056605.405 \\
20 & & 28682631948378196.60 \\
\hline
\end{tabular}


TABle 13. Polynomial Coefficients of Maclaurin Series Expansion of $B^{*}$. The terms up to $j=5$ and $j=13$ are enough for the single and double precision computations, respectively.

\begin{tabular}{rrr}
\hline$j$ & $B_{X}^{*}$ & $B_{0}^{*}$ \\
\hline 0 & 0 & +1 \\
1 & $-1 / 4$ & $-1 / 4$ \\
2 & $-1 / 32$ & $+3 / 64$ \\
3 & $-3 / 256$ & $+3 / 128$ \\
4 & $-25 / 4096$ & $+665 / 49152$ \\
5 & $-245 / 65536$ & $+3437 / 393216$ \\
6 & $-1323 / 524288$ & $+15981 / 2621440$ \\
7 & $-7623 / 4194304$ & $+188287 / 41943040$ \\
8 & $-184041 / 134217728$ & $+1293347777 / 37580963840$ \\
9 & $-4601025 / 4294967296$ & $+327273375 / 120259084288$ \\
10 & $-29548805 / 34359738368$ & $+19096474969 / 8658654068736$ \\
11 & $-193947611 / 274877906944$ & $+631505527133 / 346346162749440$ \\
12 & $-2591845347 / 4398046511104$ & $+2224154230753 / 1451355348664320$ \\
13 & $-35156056117 / 70368744177664$ & $+181962561086453 / 139330113471774720$ \\
\hline
\end{tabular}

TABle 14. Polynomial Coefficients of Maclaurin Series Expansion of $D^{*}$. The terms up to $j=5$ and $j=12$ are enough for the single and double precision computations, respectively.

\begin{tabular}{rrr}
\hline$j$ & $D_{X}^{*}$ & $D_{0}^{*}$ \\
\hline 0 & $+1 / 2$ & -1 \\
1 & $-1 / 8$ & 0 \\
2 & $-3 / 128$ & $+5 / 128$ \\
3 & $-5 / 512$ & $+31 / 1536$ \\
4 & $-175 / 32768$ & $+2365 / 196608$ \\
5 & $-441 / 131072$ & $+10409 / 1310720$ \\
6 & $-4851 / 2097152$ & $+117929 / 20971520$ \\
7 & $-14157 / 8388608$ & $+2458621 / 587202560$ \\
8 & $-2760615 / 2147483648$ & $+194646309 / 60129542144$ \\
9 & $-8690825 / 8589934592$ & $+5577961675 / 2164663517184$ \\
10 & $-112285459 / 137438953472$ & $+363577654297 / 173173081374720$ \\
11 & $-370263621 / 549755813888$ & $+632563423193 / 362838837166080$ \\
12 & $-19870814327 / 35184372088832$ & $+102453646108723 / 69665056735887360$ \\
\hline
\end{tabular}


2.2. Case of large parameter. If $0.9<m<1$, we first evaluate two modifications of the integrals:

$$
B^{*}(m) \equiv m B(m), \quad D^{*}(m) \equiv m D(m),
$$

by using the definition of Jacobi's nome and Legendre's relation. Then we compute $B(m)$ and $D(m)$ as

$$
B(m)=\frac{B^{*}(m)}{m}, D(m)=\frac{D^{*}(m)}{m} .
$$

Let us explain the details to compute $B^{*}(m)$ and $D^{*}(m)$. We begin with Jacobi's nome $q(m)$. It is defined as a function of $m$ by way of $K(m)$ as

$$
q(m) \equiv \exp \left(\frac{-\pi K(1-m)}{K(m)}\right)
$$

Refer to BF1050.01. By solving this definition with respect to $K(1-m)$ and changing the variable from $m$ to $m_{c}=1-m$, we obtain an expression of $K(m)$ in terms of $q\left(m_{c}\right)$ and $K\left(m_{c}\right)$ as

$$
K(m)=\left(\frac{K\left(m_{c}\right)}{\pi}\right)\left[-\log q\left(m_{c}\right)\right] .
$$

The Maclaurin series expansion of $q(m)$ is given as

$$
q(m)=\frac{m}{16}\left(1+\frac{m}{2}+\cdots\right) .
$$

Refer to BF901.00. Then we split the right-hand side of equation (2.5) into the sum of regular and logarithmically singular parts as

$$
K(m)=K_{0}+K_{X} X
$$

where

$$
K_{0} \equiv \frac{K\left(m_{c}\right)}{\pi}\left[-\log \left(\frac{16 q\left(m_{c}\right)}{m_{c}}\right)\right], K_{X} \equiv \frac{K\left(m_{c}\right)}{\pi}, \quad X \equiv-\log \left(\frac{m_{c}}{16}\right) .
$$

Both $K_{0}$ and $K_{X}$ are regular around $m_{c}=0$ as

$$
K_{0} \approx-\left(\frac{m_{c}}{4}+\frac{21 m_{c}^{2}}{128}\right), K_{X} \approx \frac{1}{2}+\frac{m_{c}}{8} .
$$

Meanwhile Legendre's relation is a formula on $K(m), K\left(m_{c}\right), E(m)$, and $E\left(m_{c}\right)$. It is expressed as

$$
E(m) K\left(m_{c}\right)+K(m) E\left(m_{c}\right)-K(m) K\left(m_{c}\right)=\frac{\pi}{2} .
$$

Refer to BF110.10. We obtain an expression of $E(m)$ from this as

$$
E(m)=\left[1-\left(\frac{E\left(m_{c}\right)}{K\left(m_{c}\right)}\right)\right] K(m)+\frac{\pi}{2 K\left(m_{c}\right)} .
$$

Substitute the expression of $K(\mathrm{~m})$ provided in equation (2.7) into this. Then we obtain a similar expression of $E(m)$ in terms of $X$ as

$$
E(m)=E_{0}+E_{X} X,
$$

where

$$
E_{0} \equiv \frac{\pi}{2 K\left(m_{c}\right)}+\left[1-\left(\frac{E\left(m_{c}\right)}{K\left(m_{c}\right)}\right)\right] K_{0}, \quad E_{X} \equiv\left[1-\left(\frac{E\left(m_{c}\right)}{K\left(m_{c}\right)}\right)\right] K_{X}
$$


Again, both $E_{0}$ and $E_{X}$ are regular around $m_{c}=0$ as

$$
E_{0} \approx 1-\frac{m_{c}}{4}, E_{X} \approx \frac{m_{c}}{4}+\frac{3 m_{c}^{2}}{32} .
$$

The expressions of the complete integrals using the logarithm when $m \sim 1$ have been well known from early days. Refer to Article 78 of [9], though the way of derivation is quite different. Later Hastings adopted them as a base to obtain the efficient approximations of $K(m)$ and $E(m)$ in the early days of modern computers 20. He used $Y \equiv-\log m_{c}$ as the singular variable as

$$
K(m)=K_{1}+K_{X} Y, \quad E(m)=E_{1}+E_{X} Y .
$$

Then he derived the Chebyshev polynomial approximation of $K_{X}, E_{X}$, and the new coefficients

$$
K_{1} \equiv K_{0}+K_{X} \log 16, \quad E_{1} \equiv E_{0}+E_{X} \log 16,
$$

in the domain $0 \leq m_{c}<1$. This is in order to construct a uniformly approximating formulation in low precision purposes [1. This pioneer work was extended by Cody to the case of higher precisions [10, 11, 12. Let us go further. We obtain similar expressions of $B^{*}(m)$ and $D^{*}(m)$ from equations (2.2), (2.7), and (2.12) as

$$
B^{*}(m)=B_{0}^{*}+B_{X}^{*} X, \quad D^{*}(m)=D_{0}^{*}+D_{X}^{*} X,
$$

where $B_{0}^{*}, B_{X}^{*}, D_{0}^{*}$, and $D_{X}^{*}$ are defined in terms of $K_{0}, K_{X}, E_{0}$, and $E_{X}$ as

$$
B_{0}^{*} \equiv E_{0}-m_{c} K_{0}, \quad B_{X}^{*} \equiv E_{X}-m_{c} K_{X}, \quad D_{0}^{*} \equiv K_{0}-E_{0}, \quad D_{X}^{*} \equiv K_{X}-E_{X} .
$$

They are all regular around $m_{c}=0$ as

$$
\begin{aligned}
& B_{0}^{*} \approx 1-\frac{m_{c}}{4}, B_{X}^{*} \approx-\left(\frac{m_{c}}{4}+\frac{m_{c}^{2}}{32}\right), \\
& D_{0}^{*} \approx-\left(1-\frac{51 m_{c}^{2}}{128}\right), D_{X}^{*} \approx \frac{1}{2}-\frac{m_{c}}{8} .
\end{aligned}
$$

Some low order coefficients of these approximate polynomials are listed in Tables 13 and 14. They are directly obtained by using Mathematica [22] with a command such as

$$
\text { Series[(EllipticE }[\mathrm{x}]-\mathrm{x} \text { EllipticK }[\mathrm{x}]) / \mathrm{Pi},\{\mathrm{x}, 0,20\}]
$$

This gives the coefficients of $B_{X}^{*}$ in Table 13, Numerical comparison with the original definitions reveal that the necessary minimum order of the polynomials is 5 and 13 or 12 in the single and the double precision environments, respectively. Let us summarize the procedure when $0.9<m<1$. We first compute $B_{0}^{*}, B_{X}^{*}$, $D_{0}^{*}$, and $D_{X}^{*}$ by their approximate polynomials in terms of $m_{c}$, next evaluate $X$ by calling a logarithm function once, then calculate $B^{*}(m)$ and $D^{*}(m)$, and finally obtain $B(m)$ and $D(m)$ by a simple division by $m$.

2.3. Cost and performance. We will compare the computational cost and performance of the new method with the existing ones. Let us examine the computing precision first. Figures 3 and 4 already illustrate the comparison of relative errors of $B(m)$ and $D(m)$, respectively. Obviously, the new method is the most precise. We prepared Figure 5 in order to see the error distribution of the new method integral by integral. The figure shows that the relative errors of all four complete elliptic integrals are less than the machine epsilon in the single precision environment. A 
Relative Errors of New Procedure: Single Precision

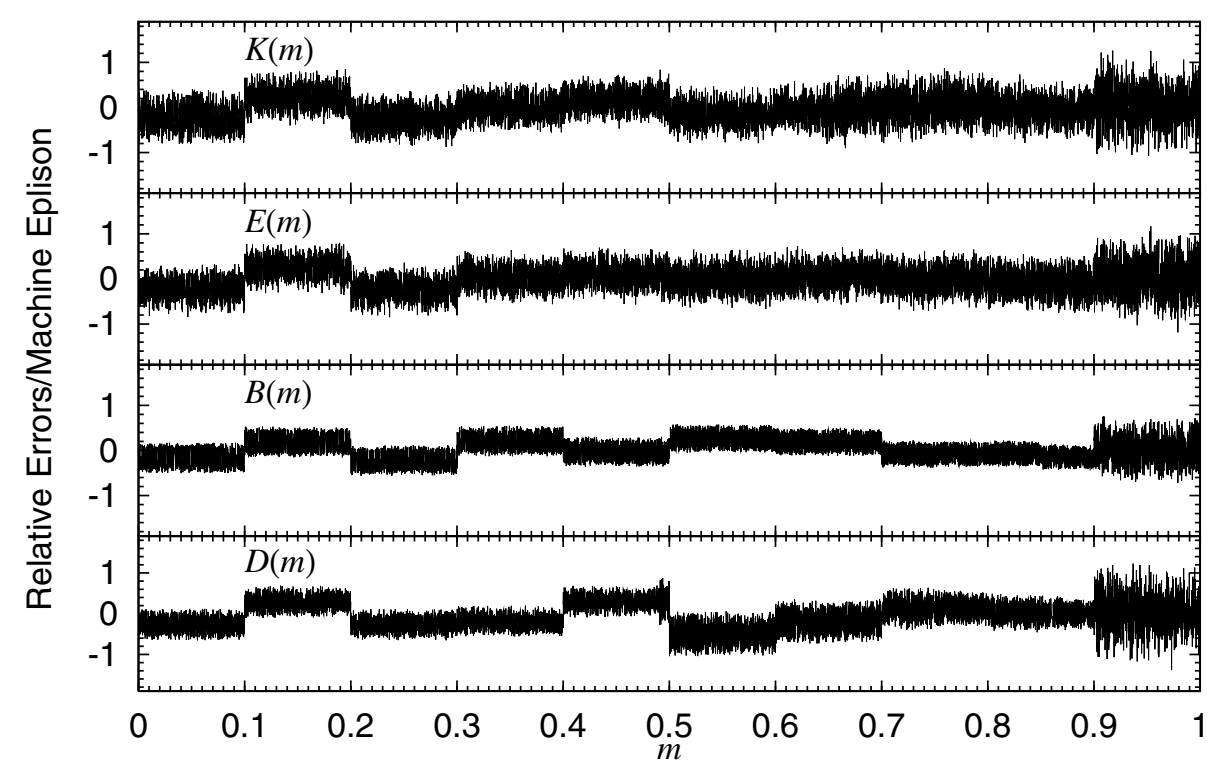

Figure 5. Relative Errors of Four Complete Elliptic Integrals Computed by New Procedure in Single Precision.

similar plot confirms that the maximum relative errors in the double precision environment are around 3 machine epsilons in the region $0.9<m<1$. The comparison in computational speed is already given in Table1. The new method calculates two polynomials of the orders 5 to 10 and 11 to 20 in the single and double precision environment, respectively. When $m>0.9$, it calls one logarithm and one division in addition. Thus the computational labor is much smaller than those of cel2, $R_{F}$, or $R_{D}$. This was easily seen in Table 1 .

\section{Conclusion}

By adopting the same approach when we developed a fast method to compute $K(m)$ and/or $E(m)$ in Paper I [17, we created a new method to calculate the auxiliary complete elliptic integrals $B(m)$ and $D(m)$. The core technique is the combination of the Taylor series expansions of the integrals, the definition of Jacobi's nome, and Legendre's relation. The new method is significantly more precise than Bulirsch's cel2 and Carlson's $R_{F}$ and $R_{D}$ in the sense that the magnitude of relative errors is less than 1-3 machine epsilons. This is mainly due to the simplicity of the algorithm and the small number of arithmetic operations required. The new method needs the evaluation of one or two polynomials of the order 5-20 by Horner's method and a call of the logarithm function provided by a standard mathematical library when $m>0.9$. Thanks to the effectiveness of the policy of divide-and-rule, the new procedure is drastically faster than the existing procedures. It runs 4.8 times faster than cel2 and 16.8 times faster than the pair of $R_{F}$ and $R_{D}$ in the double precision computation. These acceleration factors change as 5.6 and 16.4 in the 
single precision environment. The obtained procedure will be useful in developing an efficient method to compute the auxiliary incomplete elliptic integrals $B(\varphi \mid m)$ and $D(\varphi \mid m)$. Finally, we caution the readers that the new method is tailor-made and not scalable. All its parameters including the choice of subdomains, the orders of truncated polynomials, and the Taylor series coefficients have to be adapted for specific numeric processors with a higher precision as the extended precision expressed as real $* 10$ in terms of Fortran or for higher precision environments as that of quadruple $(\mathrm{real} * 16)$ precision. Here we presented the results only for the single $($ real $* 4)$ and the double $($ real $* 8)$ precision environments. Also, the new procedure assumes the availability of a mathematical library of the logarithmic function. Meanwhile Bulirsch's cel2 is scalable. One has only to prepare an approximation of $\pi$ constant. Also, it requires only the square root function apart from standard arithmetics operations. The Fortran routine of the new procedure elbd is available from the author upon request.

\section{REFERENCES}

1. Abramowitz, M., \& Stegun, I.A. (eds), Handbook of Mathematical Functions with Formulas, Graphs, and Mathematical Tables, Chapter 17. National Bureau of Standards, Washington (1964)

2. Bulirsch, R., Numerical Computation of Elliptic Integrals and Elliptic Functions, Numer. Math., 7, 78-90 (1965a) MR0175284 (30:5469)

3. Bulirsch, R., Numerical Computation of Elliptic Integrals and Elliptic Functions II, Numer. Math., 7, 353-354 (1965b) MR0185802 (32:3262)

4. Bulirsch, R., An Extension of the Bartky-Transformation to Incomplete Elliptic Integrals of the Third Kind, Numer. Math., 13, 266-284 (1969a) MR0247734(40:997)

5. Bulirsch, R., Numerical Computation of Elliptic Integrals and Elliptic Functions III, Numer. Math., 13, 305-315 (1969b) MR0247735 (40:998)

6. Byrd, P.F., \& Friedman, M.D., Handbook on Elliptic Integrals for Engineers and Physicists, 2nd ed. Springer-Verlag, Berlin (1971) MR.0060642 (15:702a)

7. Carlson, B.C., Computing Elliptic Integrals by Duplication, Numer. Math., 33, 1-16 (1979) MR:545738(80h:65008)

8. Carlson, B.C., \& Notis, E.M., Algorithm 577. Algorithms for Incomplete Elliptic Integrals, ACM Trans. Math. Software, 7, 398-403 (1981)

9. Cayley, A., An Elementary Treatise on Elliptic Functions, 2nd ed., George Bell and Sons, Cambridge (1895) MR0124532(23:A1844)

10. Cody, W.J., Chebyshev Approximations for the Complete Elliptic Integrals K and E, Math. Comp., 19, 105-112 (1965a) MR0171370(30:1601)

11. Cody, W.J., Chebyshev Polynomial Expansions of Complete Elliptic Integrals K and E, Math. Comp., 19, 249-259 (1965b) MR0178563 (31:2820)

12. Cody, W.J., Corrigenda: Chebyshev Approximations for the Complete Elliptic Integrals K and E, Math. Comp., 20, 207 (1966) MR0171370 (30:1601)

13. Fukushima, T., Simple, Regular, and Efficient Numerical Integration of Rotational Motion, Astron. J., 135, 2298-2322 (2008a)

14. Fukushima, T., Gaussian Element Formulation of Short-Axis-Mode Rotation of a Rigid Body, Astron. J., 136, 649-653 (2008b)

15. Fukushima, T., Canonical and Universal Elements of Rotational Motion of Triaxial Rigid Body, Astron. J., 136, 1728-1735 (2008c)

16. Fukushima, T., Fast Computation of Jacobian Elliptic Functions and Incomplete Elliptic Integrals for Constant Values of Elliptic Parameter and Elliptic Characteristic, Celest. Mech. Dyn. Astron., 105, 245-260 (2009a) MR2551836

17. Fukushima, T., Fast Computation of Complete Elliptic Integrals and Jacobian Elliptic Functions, Celest. Mech. Dyn. Astron., 105, 305-328 (2009b) MR2559416

18. Fukushima, T., Fast Computation of Incomplete Elliptic Integral of First Kind by Half Argument Transformation, Numer. Math., 116, 687-719 (2010) 
19. Fukushima, T., \& Ishizaki, H., Numerical Computation of Incomplete Elliptic Integrals of a General Form, Celest. Mech. Dyn. Astron., 59, 237-251 (1994) MR.1285916 (95c:65041)

20. Hastings, C. Jr., Approximations for Digital Computers, Princeton Univ. Press, Princeton (1955) MR0068915 (16:963e)

21. Innes, R.T.A., Jacobi's Nome (q) in Astronomical Formulae with Numerical Tables, M.N.R.A.S., 62, 494-503 (1902)

22. Wolfram, S., The Mathematica Book, 5th ed., Wolfram Research Inc./Cambridge Univ. Press, Cambridge (2003) MR1721106(2000h:68001)

National Astronomical Observatory of Japan, 2-21-1, Ohsawa, Mitaka, Tokyo 1818588, JAPAN

E-mail address: Toshio.Fukushima@nao.ac.jp 